\title{
NOTE
}

\section{Hepatic Function and Lipid Metabolism Are Modulated by Short-term Feeding of Cholesterol Oxidation Products in Rats}

\author{
Tasuku Sasaki ${ }^{1}$, Youichi Fujikane ${ }^{1}$, Yamato Ogino ${ }^{1}$, Kyoichi Osada $^{1,2 *}$ and \\ Michihiro Sugano ${ }^{3}$ \\ ${ }^{1}$ Faculty of Agriculture and Life Science, Hirosaki University (Hirosaki, Aomori 036-8561, JAPAN) \\ ${ }^{2}$ Department of Bioscience, Biotechnology and Agrochemistry, Meiji University (1-1-1 Higashimita, Tama-ku, Kawasaki, Kanagawa 214-8571, \\ JAPAN) \\ ${ }^{3}$ Faculty of Agriculture, Kyushu University (6-10-1 Hakozaki, Higashi-ku, Fukuoka 821-8581, JAPAN)
}

\begin{abstract}
Dietary cholesterol oxidation products (COPs) modulate various metabolic processes, particularly lipid metabolism. In this study, we observed that dietary COPs perturbed hepatic function, linoleic acid desaturation, and cholesterol catabolism in rats that were fed with diets containing $0.5 \%$ COPs for a short duration (7 days). The rats (age, 8 weeks) were fed American Institute of Nutrition (AIN)-purified diets containing $0.5 \%$ cholesterol or $0.5 \%$ COPs for 7 days. The glutamic oxaloacetic transaminase (GOT), glutamic pyruvic transaminase (GPT), and lactate dehydrogenase (LDH) levels were significantly high in rats fed with dietary COPs, but no such increase was observed in rats fed with dietary cholesterol, thereby indicating that dietary COPs may impair the hepatic function. The mRNA expression levels of $\Delta 6$ desaturase in the liver were significantly increased by dietary COPs, while these levels were significantly decreased by dietary cholesterol. However, the mRNA expression level of cholesterol 7a-hydroxylase (CYP7A1) in the liver was significantly decreased by dietary COPs and significantly increased by dietary cholesterol. Therefore, dietary COPs may modulate lipid metabolic processes such as linoleic acid desaturation and cholesterol catabolism even when they are consumed for a short duration. Hence, processed animal foods containing COPs should be consumed with caution.
\end{abstract}

Key words: cholesterol oxidation products, cholesterol, CYP7A1, $\Delta 6$ desaturase, hepatic function, linoleic acid

\section{INTRODUCTION}

In recent years, sterol oxidation products in processed foods have become a major concern because these oxycholesterols exert adverse toxic effects such as mutagenicity ${ }^{1}$, atherogenicity $^{2)}$, and immunosuppressive activity ${ }^{3}$. Cholesterol readily undergoes oxidative degradation similar to that seen in polyunsaturated fatty acids. Substantial amounts of cholesterol oxidation products (COPs) have been detected in a variety of processed foods ${ }^{4)}$ and fast foods $s^{5}$. Dietary COPs specifically perturbed growth, cholesterol metabolism, linoleic acid desaturation, and age-related changes in the lipid metabolism of rats fed with COPs for 3-4 weeks ${ }^{6}$. Moreover, dietary COPs specifically modu- late hepatic cholesterol $7 \alpha$-hydroxylase (CYP7A1) and $\Delta 6$ desaturase activities. Thus, dietary COPs exert biologically deleterious effects on lipid metabolism. However, the effects of dietary COPs on the expressions of genes encoding hepatic CYP7A1 and $\Delta 6$ desaturase have not been elucidated. Although experiments have shown that rats fed with

Abbreviations: AIN, American Institute of Nutrition; COPs, cholesterol oxidation products; CYP7A1, cholesterol $7 \alpha$-hydroxylase; DTT, dithiothreitol; GAPDH, Glyceraldehyde 3-phosphate dehydrogenase; GOT, Glutamic Oxaloacetic Transaminase; GPT, Glutamic Pyruvic Transaminase; HMG-CoA, 3-hydroxy-3-methylglutaryl Coenzyme A; LDH, Lactate Dehydrogenase; SREBP, Sterol Regulatory Element Binding Protein.

\footnotetext{
*Correspondence to: Kyoichi Osada, Laboratory of Food Hygiene, Department of Bioscience, Biotechnology and Agrochemistry, Meiji University, 1-1-1 Higashimita, Tama-ku, Kawasaki, Kanagawa 214-8571, JAPAN.

E-mail: kyochi@isc.meiji.ac.jp

Accepted March 15, 2010 (received for review January 8, 2010)

Journal of Oleo Science ISSN 1345-8957 print / ISSN 1347-3352 online

http://www.jstage.jst.go.jp/browse/jos/
} 
COPs for 3-4 weeks developed liver atrophy, the effect of COPs on hepatic function has not been completely understood up to now ${ }^{7}$. Therefore, the objective of this study is to investigate the possible mechanism underlying the deleterious effect of short-term (7 days) dietary intake of COPs on the hepatic function, cholesterol catabolism, fatty acid metabolism, and hepatic CYP7A1 and $\Delta 6$ desaturase gene expressions in rats.

\section{EXPERIMENTAL}

\subsection{Animal experiments}

Male Sprague-Dawley rats (age, 7 weeks; CLEA Japan Co., Japan) were housed individually in a room with controlled temperature $\left(20-23^{\circ} \mathrm{C}\right)$ and light $(0800-2000 \mathrm{~h})$ conditions. The rats were allowed to acclimatize to these conditions for 1 week, and then, they were divided into 3 groups of 5 or 6 ; one group was fed a cholesterol-free diet (Control group), the second group was fed a diet containing $0.5 \%$ cholesterol (Chol group), and the third group was fed a diet containing $0.5 \%$ cholesterol oxidation products (COPs) mixture(COP group). All the 3 groups were fed with the same quantity of food. The diets were prepared according to the recommendations of the American Institute of Nutrition ${ }^{8)}$ and contained the following components (percent by weight): casein, 20\%; safflower oil(Nissin Oilio Co. Ltd.), 5\%; mineral mixture (AIN93) , 3.5\%; vitamin mixture (AIN93) , 1\%; choline bitartrate, $0.25 \%$; L-cystine, $0.3 \%$; cellulose, $5.0 \%$; cornstarch, $41.7486 \%$; $\alpha$-cornstarch, $13.2 \%$; tert-butylhydroquinone, $0.0014 \%$; and sucrose to $10 \%$. Cornstarch was replaced by cholesterol $(0.5 \%)$ and COPs mixture $(0.5 \%)$ in the diets of the Chol and COP groups, respectively.

After feeding for 7 days, the rats were lightly anesthetized with diethyl ether. Then, they were bled from the abdominal aorta and the liver was quickly excised. The blood was allowed to clot at room temperature and the serum was separated by centrifugation. The serum samples were maintained at $-80^{\circ} \mathrm{C}$ until analyses. The animal experiments were conducted according to the guidelines provided by the ethical committee of experimental animal care at Hirosaki University.

\subsection{Preparation of COPs}

The COPs mixture was prepared from pure cholesterol according to the protocol described in a previous report ${ }^{6)}$. The COPs mixture contained the following components (percent by weight) : cholesterol, $9.5 \% ; 7 \alpha$-hydroxycholesterol, $2.2 \%$; $7 \beta$-hydroxycholesterol, $13.5 \%$; $5 \beta$-epoxycholesterol, $12.5 \%$; $5 \alpha$-epoxycholesterol, $12.5 \% ; 7$-ketocholesterol, 15.1\%; 25-hydroxycholesterol, 0.7\%; and unidentified oxidized cholesterols, $42.1 \%$.

\subsection{Analysis of the hepatic function parameters and lipids}

The GOT, GPT, and LDH activities in the serum samples were measured using the commercial kit (Wako Pure Chemical Industries Ltd., Tokyo, Japan). The serum and liver lipids were isolated using the protocol described by Folch et $a l .{ }^{9)}$. The levels of cholesterol and triglycerides and the fatty acid compositions of the liver phospholipids and serum cholesteryl esters were measured according to the protocol described in a previous report ${ }^{3)}$.

\subsection{RNA extraction}

Total RNA was extracted from the rat hepatic cells using EZNA Total RNA Miniprep Kit(Omega Bio-tek Inc., GA). The RNA concentration was determined by measuring the absorbance at $260 \mathrm{~nm}$ in a UV photometer. The ratios of the absorbances $(260 / 280 \mathrm{~nm})$ of all the preparations were between 1.8 and 2.0. The RNA sample aliquots were subjected to electrophoresis in a $1.4 \%$ agarose-formaldehyde gel to verify RNA integrity. The RNA concentrations were adjusted to $1 \mu \mathrm{g} / \mu \mathrm{L}$ using the UV photometer and the aliquots were stored at $-80^{\circ} \mathrm{C}$.

\subsection{Oligonucleotide primer sequences}

The primers for the rat genes $\Delta 6$ desaturase and CYP7A1 were designed by Primer 3 (http://www-genome.wi.mit. cdu/cgi-bin/primer/primer3_www.cgi). The primers synthesized by Hokkaido System Science Co. Ltd., (Sapporo Japan)were designed to flank known or putative introns, thereby preventing the amplification of any contaminating genomic DNA. Each primer sequence was as follows: Glyceraldehyde 3-phosphate dehydrogenase (GAPDH, Gene ID: 24383), forward: 5' -CTCATGACCACAGTCCATGC-3' ; reverse: 5' -TTCAGCTCTGGGATGACCTT-3' ; $\Delta 6$ desaturase (Gene ID: 83512), forward: 5' -ATGTGCCCTACAACCAG-3' ; reverse: 5' -TGTGACCCACACAAACCAGT-3' ; and CYP7A1 (Gene ID: 25428), forward: 5' -GCCGTCCAAGAAATCAAGCAG-3' ; reverse: 5' -TGTGGGCAGCGAGAACAAAGT-3' .

\subsection{Real-time quantitative polymerase chain reaction(RT- PCR)}

One microgram of total RNA, in a $10 \mu$ mixture consisting of SuperScript III reverse transcriptase (Invitrogen, CA), 40 U Rnase OUT(Rnase inhibitor), $10 \times \mathrm{RT}$ buffer (200 mM Tris-HCl(pH8.4)), $500 \mathrm{mM} \mathrm{KCl),} 25 \mathrm{mM} \mathrm{MgCl}_{2}$, and $0.1 \mathrm{M}$ dithiothreitol(DTT), was reverse transcribed during incubation at $50^{\circ} \mathrm{C}$ for $50 \mathrm{~min}$. The reverse transcription was terminated by heating the mixture at $85^{\circ} \mathrm{C}$ for $5 \mathrm{~min}$ and then quickly cooling on ice. An aliquot of the cDNA samples was mixed with $10 \mu \mathrm{l} 2 \times$ DyNAmo Master Mix (FINNZYMES, Finland) in the presence of 5 pmol of each forward and reverse primer for $\Delta 6$ desaturase and CYP7A1, and then this PCR-reaction mix was subjected to PCR under standard conditions (40 cycles). As an internal 
control, the same aliquots of cDNA samples were also subjected to PCR in the presence of a second pair of primers specific to rat GAPDH RNA. The mixtures were incubated in a Chromo 4 Sequence Detection System (Bio-Rad, CA) that was programmed to conduct one cycle at $95^{\circ} \mathrm{C}$ for 10 min and thereafter, 40 cycles at $95^{\circ} \mathrm{C}$ for $10 \mathrm{~s}$ and $57^{\circ} \mathrm{C}$ for $1 \mathrm{~min}$. The results (fold changes) were expressed as relative fold by comparing the amount of RNA of the target gene to that of the GAPDH gene, which is determined by the equation $2^{-\mathrm{C}(\mathrm{t}) \text { target-C(t)GAPDH}}$

\section{RESULTS AND DISCUSSION}

Processed foods have substantial levels of COPs ${ }^{4)}$. COPs are toxic agents and modulate metabolic processes ${ }^{10)}$. Our previous experiments showed that feeding rats with dietary COPs for relatively long durations such as 3-4 weeks disturbed the lipid metabolism and caused liver atrophy ${ }^{7)}$. However, it is not clear whether short-term dietary intake of COPs causes subacute toxicity and perturbation of lipid metabolism. In this study, we examined the effects of short-term dietary intake of COPs on the hepatic function and lipid metabolism in rats.

Dietary COPs alter growth parameters such as body

Table 1 Effects of dietary COPs on liver function indices and lipid parameter in liver and serum

\begin{tabular}{|c|c|c|c|}
\hline & \multicolumn{3}{|c|}{ Group } \\
\hline & Control & Chol & COP \\
\hline \multicolumn{4}{|c|}{ Liver function indices } \\
\hline GOT & $9.41 \pm 0.79 \mathrm{a}$ & $9.55 \pm 1.27 \mathrm{a}$ & $20.24 \pm 1.00^{b}$ \\
\hline GPT & $24.07 \pm 1.62 \mathrm{a}$ & $22.75 \pm 1.42 \mathrm{a}$ & $31.97 \pm 1.95 \mathrm{~b}$ \\
\hline $\mathrm{LDH}$ & $31.51 \pm 9.92 \mathrm{ab}$ & $23.94 \pm 4.97 \mathrm{a}$ & $49.94 \pm 3.11 \mathrm{~b}$ \\
\hline \multicolumn{4}{|c|}{$\Delta 6$ desaturation index $((20: 3(n-6)+20: 4(n-6) / 18: 2(n-6))$} \\
\hline Liver PC & $2.33 \pm 0.06$ & $1.53 \pm 0.06$ & $2.31 \pm 0.09$ \\
\hline Liver PE & $4.24 \pm 0.12 \mathrm{a}$ & $3.48 \pm 0.25 \mathrm{~b}$ & $4.91 \pm 0.19 \mathrm{c}$ \\
\hline Serum PL & $0.67 \pm 0.15$ & $0.73 \pm 0.08$ & $0.73 \pm 0.04$ \\
\hline Serum CE & $2.43 \pm 0.3 \mathrm{ab}$ & $1.64 \pm 0.27 \mathrm{a}$ & $3.15 \pm 0.29 b$ \\
\hline \multicolumn{4}{|l|}{ Lipid para meter } \\
\hline \multicolumn{4}{|l|}{$\operatorname{Serum}(\mathrm{mg} / \mathrm{dL})$} \\
\hline Cholesterol & $33.4 \pm 4.8$ & $41.6 \pm 2.1$ & $35.1 \pm 3.0$ \\
\hline Triglyceride & $39.9 \pm 5.7$ & $48.1 \pm 5.4$ & $46.8 \pm 3.1$ \\
\hline \multicolumn{4}{|l|}{ Liver (mg/g) } \\
\hline Cholesterol & $2.9 \pm 0.1 \mathrm{a}$ & $11.8 \pm 1.1 \mathrm{~b}$ & $2.1 \pm 0.0 \mathrm{a}$ \\
\hline Triglyceride & $15.3 \pm 0.3 \mathrm{a}$ & $30.1 \pm 1.6^{b}$ & $12.3 \pm 0.6 \mathrm{c}$ \\
\hline
\end{tabular}

Each value is the mean $\pm \mathrm{SE}$ ( $\mathrm{n}=5$ or 6 ). Values without a common superscript in a row are significantly different $(\mathrm{P}<0.05)$. COPs, cholesterol oxidation products mixture; Control, rats fed cholesterol-free diets; Chol, rats fed $0.5 \%$ cholesterol-fed diet; COP, rats fed $0.5 \%$ COPs; GOT, glutamic oxaloacetic transaminase; GPT, glutamic pyruvic transaminase; LDH, lactate dehydrogenase; PC, phosphatidylcholine; PE, phosphatidylethanoleamine; PL. phospholipids; CE, cholesterylester. 
weight gain in previous study ${ }^{6)}$. In this study, no statistically significant differences were observed in the body weight gain among the 3 groups; however, the relative liver weight of the COP group at the time of killing was significantly higher $(3.66 \pm 0.07 \mathrm{~g} / 100 \mathrm{~g}$ body weight $)$ than those of the Control group $(3.11 \pm 0.05 \mathrm{~g} / 100 \mathrm{~g}$ body weight $)$ and the Chol group (3.35 $\pm 0.10 \mathrm{~g} / 100 \mathrm{~g}$ body weight). Therefore, the increase in the body weight may not be influenced by the short-term dietary intake of COPs in rats. The hypertrophy of the liver due to dietary COPs may be associated with the detoxification process, but we do not have an exact explanation.

The GOT, GPT, and LDH levels in the Control and Chol groups were nearly similar; however, the levels in the COP group were higher than those of the Control and Chol groups (Table 1). Similar findings were reported in a previous study in which dietary intake of COPs for 3-4 weeks caused liver atrophy in rats ${ }^{6)}$. COPs have potent cytotoxic effects $^{1)}$, and they exert subacute toxic effects in the liver and disturb the hepatic function. Therefore, dietary COPs may cause hypertrophy in first stage and then induce liver atrophy in second stage. Further studies will be required to elucidate the relationship between dietary COPs and hepatic function.

The liver cholesterol level in the Chol group was significantly higher than that in Control group in the present study. Although the COPs mixture contained a small amount of cholesterol (9.5\%), the liver cholesterol level of the COP group was lower than that of the Control group. This decrease can be attributed to the potent inhibitory role of COPs on 3-hydroxy-3-methylglutaryl Coenzyme A (HMG-CoA reductase $)^{10)}$. However, there were no statistically significant differences in the serum cholesterol level among the 3 groups. Effects of dietary COPs on serum cholesterol level may be mild in first stage. The serum triglyceride level of the Chol group was higher than that of the Control group; however, the serum triglyceride level of the COP group was significantly lower than that of the Control group. The increase in the serum triglyceride levels in the Chol group can be explained by the fact that dietary cholesterol promotes the expression of Sterol Regulatory Element Binding Protein (SREBP)-1c mRNA in the liver ${ }^{11)}$, thereby increasing the liver triglyceride level. However, dietary COPs decrease the expression of SREBP-1c mRNA in the liver, thereby inhibiting the activities of fatty acid synthetic enzymes such as fatty acid synthetase, malic enzyme, and glucose-6-phosphate dehydrogenase. Moreover, dietary COPs inhibit the absorption of dietary fat ${ }^{12)}$. The serum triglyceride levels of the Chol and COP groups were almost similar but that of the Control group was lower than those of the other 2 groups. Further studies will be required to elucidate the mechanisms underlying these inhibitory effects of COPs.

The $\Delta 6$ desaturation index [ $(20: 3(n-6)+20: 4(n-6)) / 18: 2$
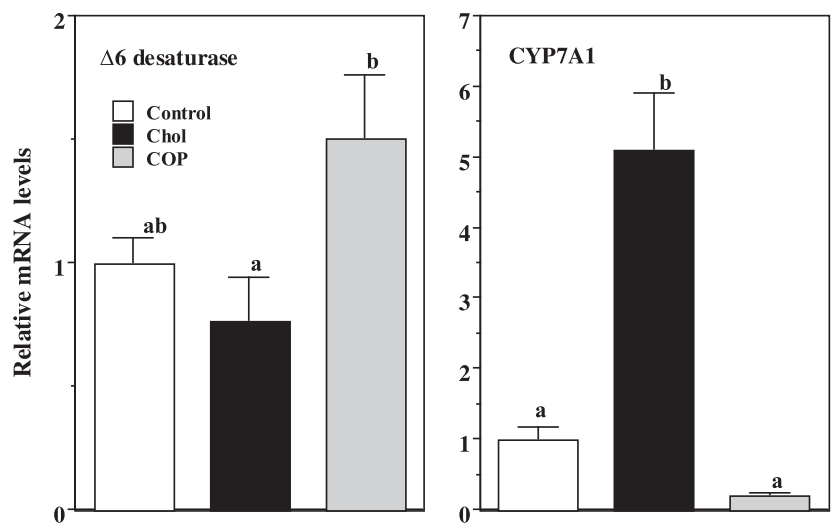

Fig. 1 Effects of Dietary COPs on Hepatic $\Delta 6$ Desaturase or CYP7A1 Gene Expression in Rats. Each value is mean $\pm \mathrm{SE}(\mathrm{n}=5$ or 6$)$. Values without a common superscript in a row are significantly different $(P<0.05)$. Control, rats fed with cholesterol-free diets; Chol, rats fed with $0.5 \%$ cholesterol diet; COP, rats fed with $0.5 \%$ cholesterol oxidation products diet.

(n-6) ] in liver phosphatidylcholine and phosphatidylethanolamine were significantly lower in the Chol group than that of the Control group. However, this index in the phosphatidylethanolamine and serum cholesterylester fraction of the COP group was significantly higher than that of the Control group, but this high index was not observed in phosphatidylcholine. Dietary COPs promoted hepatic $\Delta 6$ desaturase activity in previous studies ${ }^{6,7)}$. We observed that dietary COPs significantly increased the expression of $\Delta 6$ desaturase mRNA in the rat liver in this study (Fig. 1). Thus, dietary COPs promote linoleic acid desaturation by the modulation of the $\Delta 6$ desaturase mRNA expression in the liver. Dietary COPs may also modulate the production of eicosanoids since the supply of free substrate is one determinant of eicosanoid production. Further studies are required to elucidate the effects of COPs on arachidonic acid metabolism.

Contrary to the effect on linoleic acid metabolism, dietary COPs decreased the expression of CYP7A1 mRNA in the liver, while dietary cholesterol increased the CYP7A1 mRNA expression. Therefore, dietary COPs may inhibit cholesterol catabolism by inhibiting the CYP7A1 activity in rats. Thus, dietary COPs may perturb cholesterol homeostasis. Moreover, the absorption of lipids in the intestine may be reduced because of the inhibitory effect of dietary COPs on bile acid synthesis. 


\section{CONCLUSION}

In conclusion, our study shows the deleterious effects of short-term ( 7 days) dietary intake of COPs on the hepatic function, linoleic acid desaturation, and cholesterol catabolism in rats. Further modifications of gene expression concerning lipid metabolism can be induced by long-term dietary intake of COPs. Thus, COPs are biologically deleterious agents and may be associated with the initiation or progression of various diseases. Various levels of COPs $(0.19-4878 \mu \mathrm{g} / \mathrm{g})$ have been detected in some commonly consumed processed animal foods. We observed that dietary COPs were absorbed from intestine at approximately $30 \%$ in rats in previous study ${ }^{13)}$. Therefore, processed foods containing high level of COPs should be consumed with caution. Further, it can be considered that the consumption of foods that inhibit the absorption of COPs may also be effective to maintain a healthy body.

\section{References}

1. Sevanian, A. ; Peterson, A. R. The cytotoxic and mutagenic properties of cholesterol oxidation products. Food Chem. Toxicol. 24, 1103-1110(1986).

2. Peng, S. K.; Hu, B.; Morin, R. J. Angiotoxicity and atherogenicity of cholesterol oxides. J. Clin. Lab. Anal. 5, 144-152(1991).

3. Osada, K.; Minehira, K.; Inoue, S.; Nakamura, S.; Yamada, K.; Sugano, M. Effect of oxidized cholesterol on age-associated changes to immune parameters in spleen lymphocytes and peritoneal exudates cells derived from rats. Biosci. Biotechnol. Biochem. 64, 1047-1051 (2000).

4. Paniangvait, P.; King, A. J.; Jones, A. D.; German, B. G.
Cholesterol oxides in foods of animal origin. J. Food Sci. 60, 1159-1174(1995).

5. Lake, R. J.; Scholes, P. Consumption of cholesterol oxides from fast foods fried in beef fat in New Zealand. $J$. Am. Oil Chem. 74, 1069-1075(1997).

6. Osada, K.; Kodama, T.; Noda, S.; Yamada, K., Sugano, M. Oxidized cholesterol modulates age-related change in lipid metabolism in rats. Lipids 30, 405-413 (1995).

7. Osada, K.; Kodama, T.; Yamada, K.; Nakamura, S.; Sugano M. Dietary oxidized cholesterol modulates cholesterol metabolism and lonpleic acid desaturation in rats fed high-cholesterol diet. Lipids 33, 757-764. (1998).

8. American Institute of Nutrition. Report of the AIN Ad Hoc committee on standard for nutritional studies. $J$. Nutr. 107, 1340-1348(1997).

9. Folch, J.; Lees, M.; Sloane-Stanley, G. H. A simple method for the isolation and purification of total lipids from animal tissues. J. Biol. Chem. 226, 497-506 (1957).

10. Schroepfer, G. J. Jr. Oxysterols: Modulators of cholesterol metabolism and other processes. Physiol. Rev. 380, 61-554(2000).

11. Orolin, J.; Veoera, R.; Markova, I.; Zacharova, A.; Anzenbancher, P. Differences in hepatic expression of genes involved in lipid homeostasis between hereditary hypertriglyceridemic rats and healthy Wistar rats and in their response to dietary cholesterol. Food Chem. Toxicol. 47, 2624-2630(2009).

12. Osada, K.; Sasaki, E.; Sugano, M. Inhibition of cholesterol absorption by oxidized cholesterol in rats. Biosci. Biotechnol. Biochem. 58, 782-783(1994).

13. Osada, K.; Sasaki, E.; Sugano, M. Lymphatic absorption of oxidized cholesterol in rats. Lipids 29, 555-559 (1994). 